See discussions, stats, and author profiles for this publication at: https://www.researchgate.net/publication/328312881

\title{
Assessing implicit and explicit dementia stigma in young adults and care- workers
}

Article in Dementia - October 2018

DOI: $10.1177 / 1471301218804727$

\section{CITATIONS}

3 authors:

Alan Kane

Independent Practitioner

1 PUBLICATION 4 CITATIONS

SEE PROFILE

Michelle E. Kelly

National College of Ireland

25 PUBLICATIONS 705 CITATIONS

SEE PROFILE
193

Carol Murphy

National University of Ireland, Maynooth

49 PUBLICATIONS 345 CITATIONS

SEE PROFILE

Some of the authors of this publication are also working on these related projects:

PEAK-ABA research collaboration with Dr. Mark Dixon, SIUC View project

An examination of addicts' implicit biases towards more: A relational frame theory perspective View project 


\title{
Assessing implicit and explicit dementia stigma in young adults and care-workers
}

\author{
Alan Kane \\ National University of Ireland Galway, Ireland \\ Carol Murphy \\ National University of Ireland Maynooth, Ireland \\ Michelle Kelly (D) \\ National College of Ireland, Ireland
}

\begin{abstract}
This aim of this study was to assess implicit and self-reported stigma towards people with dementia in young adults with no contact or experience $(n=23)$, and in care-workers $(n=17$ professional dementia care-workers). Data were analysed to determine whether stigma was related to selfreported levels of depression, anxiety, stress and professional burnout. Forty participants completed the Implicit Relational Assessment Procedure and Dementia Attitudes Scale. The Depression Anxiety and Stress Scale and Maslach Burnout Inventory were used to measure depression, anxiety, stress and professional burnout. The young adult group showed statistically significant levels of dementia stigma (on the two "dementia" trial-types, $p=.027$ and $p=.030$ ). Statistical analyses showed more dementia-positive attitudes in care-workers compared to young adults on the Implicit Relational Assessment Procedure and the Dementia Attitudes Scale (both p's=.02I). Spearman's Rho correlations tests showed that for the care-givers, higher levels of burn-out were associated with more negative attitudes towards people with dementia on both of the Dementia Attitudes Scale subscales (social comfort $p<.00 \mathrm{I}$ and dementia knowledge $p=.005$ ). The results support prior research showing that experience with a stigmatised group can lower stigma and demonstrate the importance of providing a supportive work environment to mitigate burnout.
\end{abstract}

\section{Keywords}

dementia, Alzheimer's disease, stigma, implicit cognition, professional burnout 
Dementia is a progressive neurodegenerative condition characterised by deterioration in cognitive functioning across a number of domains including memory, executive function and attention (Greene, Hodges, \& Baddeley, 1995) and is often associated with behavioural and psychological symptoms including depression, aggression and wandering (Grand, Caspar, \& MacDonald, 2011). Research has focused on neurological, contextual and genetic factors associated with the onset of dementia; but no definitive cause has been identified (Cahill, O'Shea, \& Pierce, 2012). Although treatments with medication can improve cognitive function and behaviour (Winslow, Onysko, Stob, \& Hazlewood, 2011), a cure is not forthcoming. There were an estimated 50 million people worldwide living with dementia in 2017. This figure is expected to rise to 75 million by 2030 (in Alzhiemers report, 2015; Prince, Wimo, Guerchet, Ali, Wu, \& Prina, 2015). The risk of dementia rises significantly with age, with about $70 \%$ of all dementia cases occurring in people over 75 years old (Fratiglioni et al., 2000). The increasing numbers of people with dementia living in the community coupled with relatively limited treatment options is likely to have significant implications for community support resources, including the need for provision of supportive environments and dementia friendly societies.

\section{Stigma towards people with dementia}

Stigma refers to negative attitudes and biases about individuals perceived as being different to oneself, possibly due to a diagnosis, condition or illness (Dinos, Stevens, Serfaty, Weich, \& King, 2004). Stigmatising attitudes can lead people to respond negatively to, be prejudiced towards, avoid, or even be fearful of others (Corrigan \& Watson, 2002). In research analysing public attitudes towards people with dementia, only $4 \%$ of participants believed that people with dementia were to blame for their behaviours and respondents wanted to help those with dementia, seeing them as patients that needed to be cared for (Werner \& Davidson, 2004). Although these are empathetic attitudes, viewing people with dementia as helpless and dependent may perpetuate stigma. In general, people tend to possess negative and infantilising attitudes towards those with dementia (Jorm, 2000; McParland, Devine, Innes, \& Gayle, 2012) and believe that they lack awareness (Clare, 2003). For care professionals, attitudes towards people with dementia can be positive (Kada, Nygaard, Mukesh, \& Geitung, 2009) with high levels of job satisfaction associated with more positive biases (Moyle, Murfield, Griffiths, \& Venturato, 2011). On the other hand, nursing home staff have reported feelings of frustration and anxiety towards residents with dementia that display aggressive behaviours (Draper et al., 2000). Staff can also experience high levels of stress (Rodney, 2000) which in turn may lead to negative or stigmatising attitudes (Astrom, Nilsson, Norberg, Sandman, \& Winblad, 1991). Assessing and improving attitudes towards people with dementia is of utmost importance as stigma can lead to stereotypes, prejudice, and discrimination (Corrigan \& Watson, 2002), and might have serious implications for the well-being of people with dementia and their carers.

\section{The impact of stigma}

Stigma can affect an individual on an emotional and personal level (Burgener \& Berger, 2008; Scholl \& Sabat, 2008), and can interfere with social interactions with family members 
and others. Carers of people with dementia believe that society does not want to engage with or hear about people with dementia, and this leads to feelings of desperation and a lack of support for both the person with dementia and their family (Cahill et al., 2012; Hastings, 2009). A concerning outcome of this is that often people delay seeking a diagnosis (Batsch \& Mittelman, 2012; Cahill et al., 2012; Iliffe et al., 2005). When a diagnosis is sought, there is difficulty in communications between GPs and specialists in relation to dementia detection (Ólafsdóttir, Foldevi \& Marcusson, 2001) and difficulty in delivering the diagnosis (Bamford et al., 2004; Clafferty, McCabe, \& Brown, 2001; Mitchell, 2007). This has serious implications as GPs are gatekeepers between the general public and specialised dementia care. After diagnosis, individuals can attempt to conceal their illness and fail to seek assistance (Batsch \& Mittelman, 2012) which is problematic as early diagnosis and treatment are integral for the successful implementation of interventions such as cognitive stimulation therapies (Moniz-Cook, Agar, Gibson, Win, \& Wang, 1998; Spector et al., 2003) and behavioural therapies (Teri, Logsdon, Uomoto, \& McCurry, 1997). Stigma may also contribute to feelings of anxiety or depression for the person post-diagnosis (Bender \& Cheston, 1997; Jessen et al., 2014; Katz, 1998; Lobo, Saz, Marcos, Día, \& De-la-Cámara, 1995). Benbow and Jolley (2012) have argued that stigma has a negative impact on multiple levels, including help-seeking for individuals affected, service-provision, funding, and policy decisions about resource priorities.

\section{Factors affecting stigma}

Information and level of understanding or knowledge can mediate stigmatising attitudes towards people with dementia (Batsch \& Mittelman, 2012; Cheng et al., 2011; Werner, 2005). The World Alzheimer Report 2012 attributes a lack of information and education about dementia as one of the primary causes of stigma (Batsch \& Mittelman, 2012). Cheng et al. (2011) reported that that even brief exposure to information about dementia led to a statistically significant reduction in stigma. In addition, those who were more educated and younger had more positive attitudes towards the disease than those who were not (Cheng et al., 2011). Similar findings regarding level of education and stigma were reported in a study in Brazil (Blay \& Peluso, 2010) but a recent systematic review reported conflicting evidence regarding age, showing that stigmatising attitudes are worse among younger individuals (Herrmann et al., 2017). Among professional care staff, those who had greater knowledge and received appropriate training had positive attitudes towards dementia (Moyle et al., 2011; Richardson, Kitchen, \& Livingston, 2002), although these findings are not consistent (Rüsch, Angermeyer, \& Corrigan, 2005). Factors such as depression, anxiety, stress, burnout, and lower job satisfaction can have a negative impact on the attitudes of those working in a professional capacity with stigmatised groups (Chirwa et al., 2009; Kelly \& Barnes-Holmes, 2013); although these factors have yet to be investigated in the context of dementia stigma. Research on dementia stigma is seriously lacking. A recent systematic review identified only 7 studies that investigated dementia stigma in healthcare professionals and 11 that examined the lay public (Herrmann et al., 2017). Further investigation is required to determine extant levels of stigma in specific community groups, and to examine factors that might influence stigma. This information can be used to 
provide a more solid evidence-base to inform educators, trainers, policy-makers and community care providers.

\section{Measuring dementia stigma}

To date, the assessment of dementia stigma has relied on subjective self-report measures including the Dementia Attitudes Scale (DAS; George, Stuckey, \& Whitehead, 2014; O'Connor \& McFadden, 2010; Scerri \& Scerri, 2013) and the Dementia Screening Subscale of the PRISM-PC (Boustani et al., 2008). The use of the subjective measures has resulted in conflicting findings (e.g. Moyle et al., 2011; Werner \& Davidson, 2004), resulting in a call for research that better identifies and measures stigma (Herrmann et al., 2017). A novel approach to measuring stigma might include the use of an implicit attitude measure. Implicit measures capture responses that are immediate, automatic, and less controlled than subjective responses (De Houwer, 2006; Hughes, Barnes-Holmes, \& De Houwer, 2011), and may therefore provide a more nuanced approach to measuring stigma (for more information on implicit measures, see De Houwer, 2006; Greenwald, McGhee, \& Schwartz, 1998).

The Implicit Relational Assessment Procedure (IRAP; Barnes-Holmes et al., 2006) is a computerised assessment of implicit responding that has provided the scientific community with valuable information regarding implicit attitudes towards various social groups (Barnes-Holmes, Murtagh, Barnes-Holmes, \&, Stewart, 2010; Cullen \& Barnes-Holmes, 2008; Kelly \& Barnes-Holmes, 2013; Murphy, Hussey, Barnes-Holmes, \& Kelly, 2015; Murphy, MacCarthiagh, \& Barnes-Holmes, 2014; Roddy, Stewart, \& Barnes-Holmes, 2010) including older adults (Cullen, Barnes-Holmes, Barnes-Holmes, \& Stewart, 2009). Comparisons between the IRAP and self-report measures often show that the IRAP can detect implicit biases that were not revealed by questionnaires (for a full account, see Barnes-Holmes et al., 2006; Barnes-Holmes, Barnes-Holmes, Stewart, \& Boles, 2010; Hussey, Barnes-Holmes, \& Barnes-Holmes, 2015; Nicholson \& Barnes-Holmes, 2012). Considering the sensitive nature of assessing stigma towards people with dementia, the use of an implicit measure such as this, alongside traditional questionnaires, may add to the literature by providing insights into dementia biases not previously investigated.

\section{The current study}

The current study aimed to assess self-reported and implicit dementia stigma in a sample of young adults and in care-workers employed to care for people with dementia. We wanted to determine if stigma existed in a young, educated sample; if experience working with people with dementia affected levels of stigma; and whether self-reported and implicit reports of stigma differed within groups. Measures of dementia stigma included the DAS and the IRAP. Additional outcome measures included the Depression, Anxiety and Stress Scale, DASS; Lovibond \& Lovibond, 1995) for all participants; and the Maslach Burnout Inventory (MBI; Maslach \& Jackson, 1981) for care-workers. We hypothesised based on prior research, that due to their age (18-25) young adults would show stigmatising attitudes towards people with dementia; that care-workers would hold the most positive attitudes overall due to their knowledge, education and experience; and that factors such as depression, anxiety, stress, and burnout would be associated with negative stigmatising attitudes. 


\section{Methods}

\section{Participants}

Fifty-six individuals participated in the study. Recruitment was conducted via convenience sampling. Participants were divided into two groups, young adults aged between 18 and 25 $(n=26)$ and care-workers who worked in a professional capacity with individuals with dementia $(n=30)$. Care-workers were recruited from three day-care and respite centres in the South of Ireland, were all employees of the Alzheimer's Society of Ireland, and had received dementia-specific training (experience ranged from 1 to 32 years). Younger adults were predominantly recruited from psychology lectures in Maynooth University, with a small number $(n=5)$ also recruited from localities in the Midlands and South. The young adults reported having little/no experience of people with dementia. None of the participants provided informal unpaid care to a person with dementia or had an immediate family member with dementia. For their data to be included in the study, participants were required to meet predefined pass criteria on the IRAP (Hussey et al., 2015; see procedure). Sixteen participants data were excluded as they failed the IRAP screening procedure (Figure 1). The final sample therefore included 40 participants; $n=23$ younger adults (13 males and 10 females; mean age $=21.5$; age range $=19-25)$ and $n=17$ care-workers ( 2 males and 15 females; mean age $=26.88$; age range $=19-51$ ). Thirteen care-workers were aged between 19 and 24 and had between 1 and 5 years' experience, and the remaining four were aged between 41 and 51 and had between 16 and 32 years' experience.

\section{Experimental design}

The research was conceptualised as a mixed $2 \times 4$ factorial design. For the analysis of the IRAP data, the between participant independent variable (IV) was Group, with two levels of Care-Workers and Young Adults. The within-participant repeated measures IV was IRAP trial-type, with four levels including Dementia-Positive; Dementia-Negative; Healthy-Positive; Healthy-Negative. The dependent variable (DV) was participants' IRAP $D$-scores. For the analysis of the questionnaire data, the between participant IV was Group and the within participant IV was Subscale, and the DV was the participants score on each subscale.

\section{Ethical considerations}

Ethical approval by the Ethics Committee, National University of Ireland Maynooth. All participants provided informed consent and all research procedures were conducted in accord with current ethical practices in the field of psychology. The researchers managed and stored data in accordance with the Maynooth University Data Protection Policy and the Data Protection Act.

\section{Apparatus and materials}

The research was conducted either in laboratory cubicles at Maynooth University, in participants' homes, or at the place of employment of care-workers. In each case, participants sat at a table in a quiet room free from any distractions. The IRAP programme was presented on a standard Dell laptop; and the IRAP software controlled all aspects of the 


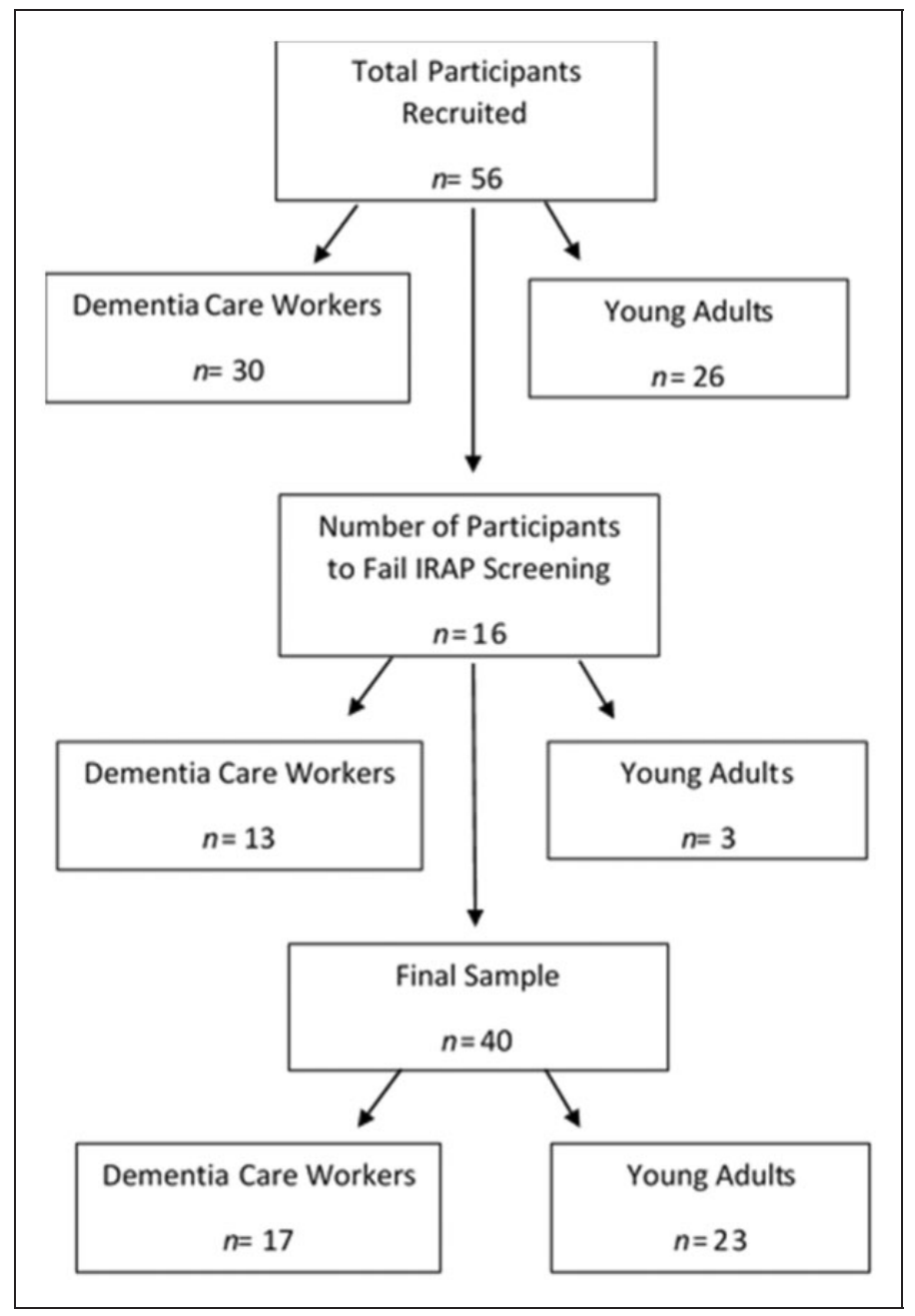

Figure I. Flow diagram showing the total number of participants recruited, the final analysable sample, and reasons for attrition.

programme presentation (IRAP software is available upon formal request to Prof. Dermot Barnes-Holmes: Dermot.Barnes-Holmes@ugent.be). The research administered the questionnaire after the IRAP was completed.

The IRAP. The IRAP stimuli are presented in Table 1. Stimuli were selected by reviewing the literature to seek out one-worded "negative" terms used towards people with dementia, such as difficult, unpleasant (Brodaty, Draper \& Low, 2003) and helpless (Werner \& Davidson, 2004). We avoided emotive terms such as poor, unfortunate or suffering. Corresponding positive terms were chosen to reflect opposite responses (e.g. incompetent/capable; irritating/enjoyable). The IRAP presented rules for participants to follow that were deemed either stereotype "consistent" or stereotype "inconsistent" based on findings of prior research on 
Table I. Stimulus arrangements and word groups presented by the IRAP.

\begin{tabular}{ll}
\hline Sample I & Sample 2 \\
\hline Capable & Incompetent \\
Able & Unable \\
Independent & Helpless \\
Pleasant & Unpleasant \\
Positive & Difficult \\
Enjoyable & Irritating \\
Targets deemed consistent with Sample I & Targets deemed consistent with Sample 2 \\
Healthy adult & Adult with dementia \\
Response Option I & Response Option 2 \\
True & False \\
\hline
\end{tabular}

IRAP: Implicit Relational Assessment Procedure.

dementia stigma (e.g. Burgener \& Berger, 2008; Scholl \& Sabat, 2008; Werner, 2005). The consistent rule stated that "Healthy adults are positive and adults with dementia are negative"; and the inconsistent rule stated that "Healthy adults are negative and adults with dementia are positive". The presentation of the consistent rule versus the inconsistent rule first was counterbalanced across participants. The IRAP presented the target stimuli at the top of the screen, the positive and negative sample stimuli in the centre of the screen; and the response options at the bottom left and right of the screen.

Dementia Attitudes Scale. The DAS (O'Connor \& McFadden, 2010) is a 20-item scale which assesses attitudes towards dementia across two factors; knowledge and beliefs about dementia, labelled "dementia knowledge" (10 questions, e.g. "People with dementia can feel when others are kind to them" and "We can do a lot now to improve the lives of people with dementia"), and behaviours and feelings of comfort around people with dementia, labelled "social comfort" (10 questions, e.g. "I feel relaxed around people with dementia" and "I cannot imagine caring for someone with dementia"). Four questions related to familiarity or behaviour $(8,10,11,20)$ while the remaining 16 questions related to stigmatising attitudes. Responses to statements were scored on a seven-point Likert scale ranging from "strongly disagree" to "strongly agree" with a possible total score range of 20-140 (five scores were reversed). Higher scores indicated more positive attitudes on both scales (scores below $80=$ dementia negative; scores above $80=$ dementia-positive). To develop the questionnaire, researchers initially conducted a qualitative analysis of structured interviews with five family caregivers, five professionals and five college students (O'Connor \& McFadden, 2010). A 46-item scale was developed, which was reduced to 20-items following principal axis factoring with certified nursing assistants $(n=302)$ and college students $(n=145)$; and confirmatory factor analysis with college students $(n=157)$. Total scale Cronbach's alphas ranged from 0.83 to 0.85 and the DAS correlated significantly with measures of stigma towards older adults and those with disabilities (O'Connor \& McFadden, 2010).

Depression, Anxiety and Stress Scale. The DASS (Lovibond \& Lovibond, 1995) is a 42-item scale which assesses the extent to which participants experience feelings of depression (e.g. "I felt sad and depressed"), anxiety ("I was aware of dryness in my mouth") or stress 
("I found myself getting agitated") within the past week. There are 14 items for each of scales of depression, anxiety and stress. Responses were scored on a four-point scale ranging from "most of the time" to "never", with a possible total score range of 42-168. Higher scores indicated lower levels of depression, anxiety and stress. The DASS has been reported to possess good reliability and validity (Crawford \& Henry, 2003; Lovibond \& Lovibond, 1995).

Maslach Burnout Inventory. The MBI (Maslach \& Jackson, 1981) is a 25-item scale which assesses professional burnout across three dimensions; "emotional exhaustion" resulting from reduced emotional resources available to the person (e.g. "I feel emotionally drained from my work", nine questions), "depersonalisation" which may lead to negative disparaging feelings towards people with dementia (e.g. "I feel I treat some recipients as if they were impersonal objects", eight questions), and reduced "personal accomplishment" resulting in a lower sense of achievement (e.g. "I have accomplished many worthwhile things in my job", eight questions) (Kokkonen, Cheston, Dallos, \& Cordet, 2014). Responses were scored on a seven-point scale ranging from "never" to "always", with a possible total score range of 7175. Higher responses for depersonalisation and emotional exhaustion scales indicated higher rates of burnout. For consistency, scores for the personal accomplishment scale were reversed so that higher scores also indicated higher levels of burnout. The MBI was valid and reliable in assessing professional burnout (Taris, Schreurs, \& Schaufeli, 1999).

\section{Procedure}

The IRAP. The IRAP was administered to all participants. The target stimulus was presented at the top of the screen (e.g. Adult with Dementia or Healthy Adult); one of the sample stimuli were presented below this (e.g. incompetent or independent), with response options "true" and "false" presented at the bottom left and right of the screen. Participants chose "true" by pressing the " $\mathrm{d}$ " key and "false" by pressing the "k" key (see Table 1). For the consistent rule, participants were required to pair "healthy adult" with positive words (healthy-positive), and "adult with dementia" with negative words (dementia-negative); for the inconsistent rule, participants were required to pair "adult with dementia" with positive words (dementia-positive), and "healthy adult" with negative words (healthy-negative).

The IRAP presented blocks of 24 trials. Each of the 12 positive and negative sample stimuli appeared once with the two target stimuli. The IRAP commenced with practice blocks designed to allow participants to reach a performance criterion prior to beginning the test phase. Participants completed a maximum of four sets of practice blocks with one consistent and one inconsistent block of trials per set. Once practice criteria were met on a set of practice blocks ( $>80 \%$ accuracy and $\leq 2000 \mathrm{~ms}$ ), the programme presented test blocks. If practice criteria were not met across four practice blocks, the programme ended and participants were excused from the experiment. Once the test phase of the IRAP commenced, participants continued until all six blocks were completed. If performance on a consistent and inconsistent block fell below $80 \%$ accuracy or exceeded $2000 \mathrm{~ms}$, the data for that participant were discarded (see Barnes-Holmes, Barnes-Holmes, et al., 2010 for a thorough description of the IRAP programme).

Questionnaire measures. Upon completion of the IRAP, the young adults completed the DAS and the DASS. The care-workers completed the DAS, the DASS, and the MBI. 


\section{Results}

\section{IRAP data}

The IRAP data were participants' response latencies across IRAP trial-blocks, defined as time in milliseconds between the onset of an IRAP trial and a correct response. The response latency data were transformed into $D$-IRAP scores using an adaptation of the Greenwald, Nosek, and Banaji (2003) D algorithm (see Barnes-Holmes, Murtagh, et al., 2010; Cullen \& Barnes-Holmes, 2008) and analysed in accordance with the most recent IRAP analysis guidelines published by Hussey et al. (2015). Positive scores on the healthy-positive and dementia-positive trial-types indicated that participants responded faster with the "True" response option. Positive scores on the healthy-negative and dementia-negative trial-types indicated that participants responded faster with the "False" response option (see Figure 2).

\section{IRAP analysis}

The overall mean $D$-IRAP scores for both groups across each trial-type are presented in Figure 2. The graph shows that the young adults responded to suggest that healthy adults are positive "healthy-positive" $t(22)=4.907, p>.001$ and are not negative "healthy-negative" $t(22)=4.090, p=.001$. The care-workers showed a similar pattern of responding but the effect was only significant for the "healthy-positive" trial-type, $t(16)=4.294, p=.001$. The graph also shows that young adults responded to suggest that adults with dementia are not positive, "dementia-positive" $t(22)=-2.371, p=.027$, and are negative "dementianegative" $t(22)=-2.326, p=.030$. The care-workers did not show significant effects on either

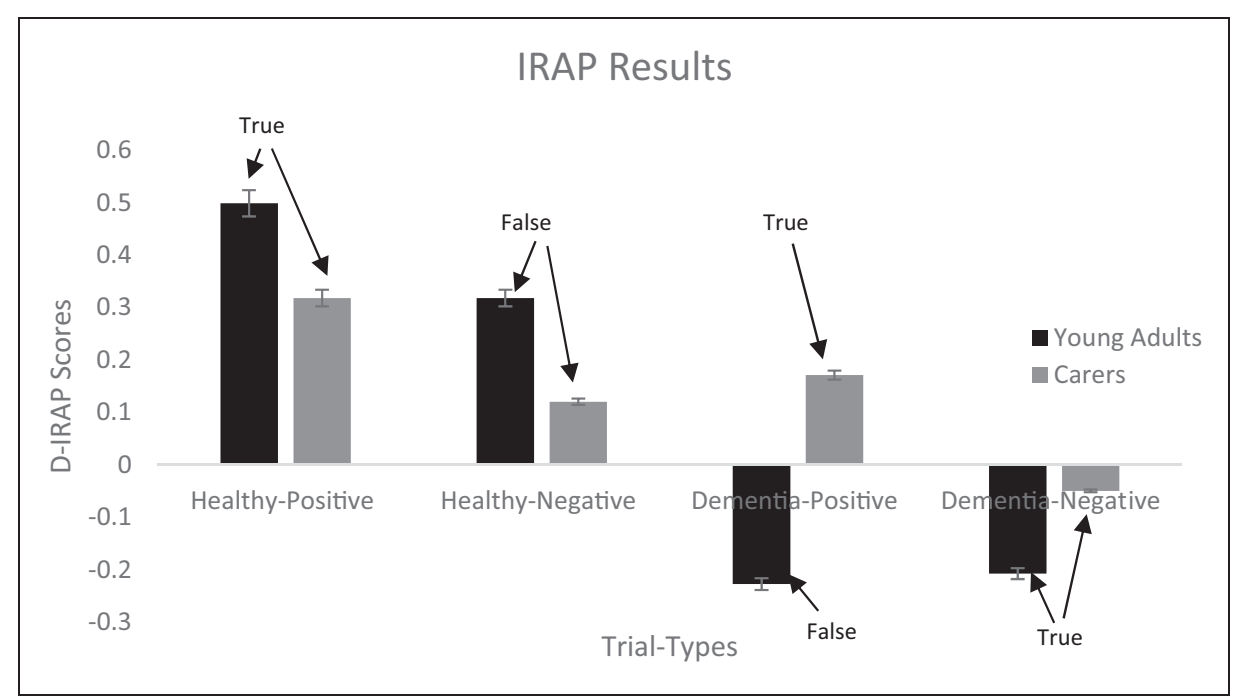

Figure 2. The mean D-IRAP scores for young adults and care-workers. The mean D-IRAP scores for young adults and care-workers. Young Adults and Care-workers showed a pro-Healthy bias, and Young Adults showed a greater anti-Dementia bias. 
of the "dementia" trial-types ("dementia-positive" $t(16)=1.797, \quad p=.091$, "dementianegative" $t(16)=-.547, p=.592)$; their responses showed a weak dementia-positive and very weak dementia-negative bias. An independent samples t-test revealed that scores on the "dementia-positive" trial-type differed significantly between groups, $t(38)=-2.878$, $p=.007$ with the young adults showing a negative bias towards people with dementia but the care-workers showing a positive bias.

A $2 \times 4$ mixed ANOVA was conducted to assess the impact of group (young adults, $n=23$ and care-workers, $n=17$ ) on $D$-IRAP scores. The within participant factor was trial-type and the between participant factor was group. The DV was $D$-IRAP score. Results showed a significant interaction effect between group and trial-type, F $(3,38)=$ 4.035, $p=0.021$, indicating that Group had an impact on trial-type scores with care-workers more likely to show positive attitudes towards people with dementia than young adults. Results also showed a significant main effect for trial-type, $\mathrm{F}(3,38)=11.526, p<0.001$. Pairwise comparisons showed that all trial-types differed significantly from one another (all $p$ 's $>0.05)$ except the "dementia-positive" and "dementia-negative" trial-types $(p=.173)$.

\section{Questionnaire analysis}

DAS. A $2 \times 2$ mixed ANOVA was conducted to assess the difference between groups on scores in the DAS subscales, social comfort and dementia knowledge. There was no significant effect for subscale, $\mathrm{F}(1,38)=.254, p=.617$, and no interaction effect, $\mathrm{F}(1,38)=.000$, $p=.997$. There was a significant main effect for group however, $\mathrm{F}(1,38)=5.821, p=.021$ showing that care-workers had significantly more dementia-positive attitudes that young adults $(M D i f f=5.013, S E=2.078)$. The mean scores of over 80 for both groups indicated overall positive attitudes towards people with dementia (see Table 2).

DASS. A $2 \times 3$ mixed ANOVA was conducted to assess the difference between groups on scores in the DASS subscales, depression, anxiety and stress. There was no significant main effect for group, $\mathrm{F}(1,38)=1.504, p=.228$ and no interaction effect between group and DASS scores, $F(2,38)=.741, p=.480$. There was a significant main effect for subscale, $F$ $(2,38)=4.009, p=.022$. Pairwise comparisons showed that scores on the stress subscale were significantly lower than on the depression and stress scales, both $p$ 's $<.05$. Overall, DASS subscale scores were within the normal range for both groups (see Table 2).

\section{Correlational analysis}

Shapiro-Wilk's tests of normality indicated that the IRAP Trial-type scores were normally distributed but one or more scales of the DAS, DASS and MBI were not; Spearman's Rho was therefore used to assess relationships between all variables (see Table 3). Most notably for the care-worker group, there were significant correlations between the personal accomplishment scale of the MBI and DAS comfort $(r=-.773, n=17, p<.001)$ and DAS knowledge $(r=-.642, n=17, \mathrm{p}=.005)$, indicating that higher levels of burn-out were associated with more negative attitudes towards people with dementia. The remaining correlations for both groups were expected as subscales of the same measures were associated. 
Table 2. Demographic information and descriptive statistics for the implicit and questionnaire measures.

\begin{tabular}{|c|c|c|c|c|c|c|}
\hline & $n$ & Range & Min & Max & Mean & SD \\
\hline \multicolumn{7}{|l|}{ Care-workers } \\
\hline Age & 17 & - & 19 & 51 & 26.88 & 11.26 \\
\hline IRAP Healthy-Positive & 17 & - & -.24 & .80 & .32 & .31 \\
\hline IRAP Healthy-Negative & 17 & - & -.59 & .78 & .12 & .42 \\
\hline IRAP Dementia-Positive & 17 & - & -.52 & .78 & .17 & .39 \\
\hline IRAP Dementia-Negative & 17 & - & -.44 & .88 & -.05 & .37 \\
\hline DAS Comfort & 17 & $10-70$ & 40 & 70 & 49.71 & 7.51 \\
\hline DAS Knowledge & 17 & $10-70$ & 41 & 70 & 50.16 & 6.95 \\
\hline DAS Total & 17 & $20-140$ & 82 & 140 & 99.76 & 13.63 \\
\hline DASS Total & 17 & $42-168$ & 68 & 168 & 136.90 & 25.69 \\
\hline MBI Total & 17 & $7-175$ & 53 & 121 & 77.11 & 19.22 \\
\hline \multicolumn{7}{|l|}{ Young adults } \\
\hline Age & 23 & - & 19 & 25 & 21.52 & 1.47 \\
\hline IRAP Healthy-Positive & 23 & - & $-.4 \mathrm{I}$ & 1.57 & .50 & .49 \\
\hline IRAP Healthy-Negative & 23 & - & -.27 & 1.53 & .32 & .37 \\
\hline IRAP Dementia-Positive & 23 & - & -1.46 & .54 & -.23 & .46 \\
\hline IRAP Dementia-Negative & 23 & - & -1.16 & .45 & -.21 & .43 \\
\hline DAS Comfort & 23 & $10-70$ & 32 & 60 & 44.69 & 6.58 \\
\hline DAS Knowledge & 23 & $10-70$ & 32 & 60 & 45.04 & 6.53 \\
\hline DAS Total & 23 & $20-140$ & 64 & 120 & 89.74 & 12.50 \\
\hline DASS Total & 23 & $42-168$ & 69 & 168 & 125.93 & 29.51 \\
\hline
\end{tabular}

IRAP: Implicit Relational Assessment Procedure; DAS: Dementia Attitudes Scale; MBI: Maslach Burnout Inventory; DASS: Depression, Anxiety and Stress Scale.

\section{Discussion}

We sought to determine whether implicit and self-reported stigma towards people with dementia existed in young adult and care-worker samples; whether the knowledge and experience of care-workers impacted levels of stigma compared to young adults; and whether burnout, depression, anxiety or stress would be related to stigma. We hypothesised that healthy-adults would be considered to be more positive than people with dementia; careworker stigma would be lower than young adults' due to care-workers' experience and training (Herrmann et al., 2017; Moyle et al., 2011) and young adults' age (Herrmann et al., 2017); and that depression, anxiety, stress and burnout would correlate with stigma (Kelly \& Barnes-Holmes, 2013). The topic of dementia stigma has not been previously examined in IRAP research and has been notably scarce among implicit research in general. This research was therefore largely exploratory.

As expected, the IRAP results showed that both groups held significant positive attitudes towards healthy adults but not towards people with dementia, while the young adults showed significant negative attitudes towards people with dementia. This supports our hypothesis that overall, adults with dementia are not considered as favourably as healthy adults; and is consistent with prior studies demonstrating the existence of dementia stigma (Batsch \& Mittelman, 2012; Cheng et al., 2011; McParland et al., 2012; Peris, Teachman, \& Nosek, 2008). Stigmatising attitudes can be manifested by individuals considering those with 


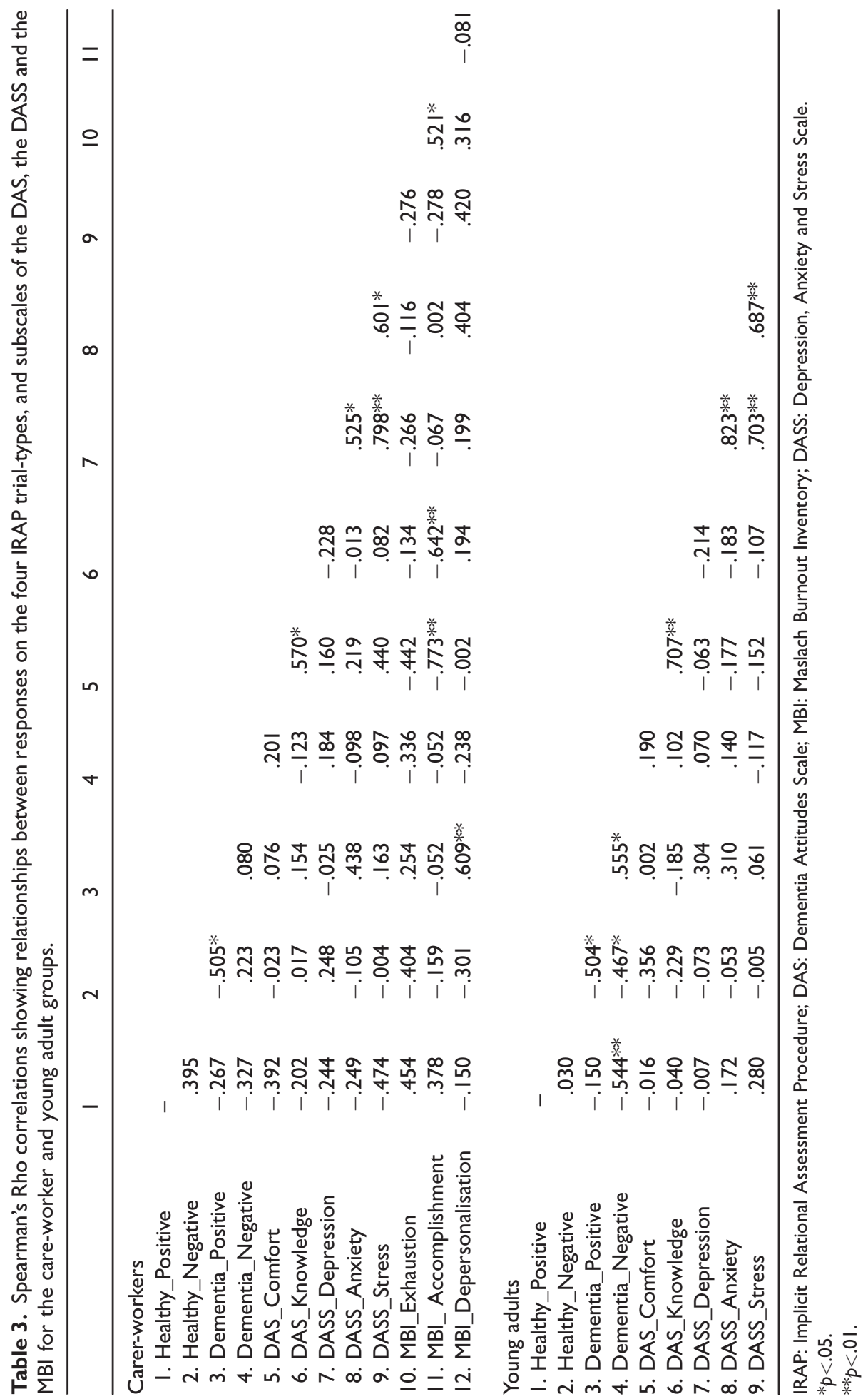


dementia as infantilised (Jorm, 2000), lacking awareness (Clare, 2003), and even potentially dangerous (Corrigan et al., 2000). Public awareness campaigns should aim to reduce the discrepancies in attitudes towards healthy adults versus those with dementia by highlighting the many cases of people that are independently residing in and contributing to their community and are "living well" with dementia.

Results from both the DAS and the IRAP showed that care-workers were significantly more likely to show positive attitudes towards people with dementia than young adults. This is consistent with prior questionnaire-based research that found that people who worked in a professional capacity with people with dementia, and who had more experience and training, showed less dementia-negative attitudes (Cheng et al., 2011; Kada et al., 2009; Moyle et al., 2011; Richardson et al., 2002). It is possible that age, as opposed to experience, may have influenced the observed effects as the age-range for care-workers was much broader than that of the younger adult sample. Conflicting evidence exists in the literature regarding the impact of age on mental health and dementia stigma, with some suggesting that increased age is associated with less stigma (Ewalds-Kvist, Högberg, \& Lützén, 2013) and others showing that younger people hold less stigmatising attitudes (Cheng et al., 2011; Park, Kim, Cho, \& Lee, 2015). In our study, four care-workers were aged over 25 (aged 41-51). Out of interest, we conducted the analyses with the four older care-workers removed and found that the effects were retained for all significant outcomes except for the DAS between group differences. This might indicate that age (as opposed to experience) impacted explicit stigma ratings, although the observed effects may still have been mediated by experience as the older care-workers had many years more experience than the younger care-workers. In any case, the numbers are too small to run any meaningful analysis but this certainly an avenue for further investigation.

We found no scale by group interactions on the DAS, suggesting that knowledge of dementia was not more influential than comfort around dementia in influencing the between-groups differences. This might indicate that either learning more about dementia or direct experience with people with dementia may be equally beneficial as interventions to reduce stigma. In implicit research, education, knowledge and training have also positively impacted attitudes towards stigmatised groups including older adults (Cullen et al., 2009) and those with mental health difficulties (Peris et al., 2008). This further reiterates the importance of dementia-specific education and training in addressing issues related to stigma.

As hypothesised, the young adults showed the most negative attitudes towards people with dementia. This is consistent with prior research in dementia (Crisp, Gelder, Goddard, \& Meltzer, 2005; Herrmann et al., 2017) and across diagnoses ranging from addiction to schizophrenia (Crisp et al., 2005), with negative attitudes even lessening as participants got older (Crisp et al., 2005). One possible explanation for greater dementia-stigma in the younger population might be a lack of exposure to dementia, and a lack of motivation to learn about the disease. This lack of information and knowledge is likely to perpetuate stigma (Batsch \& Mittelman, 2012). A second explanation may have to do with ageism. Cullen et al. (2009) reported that university students held negative biases towards older adults; and dementia is often thought of as an older person's disease. It is possible that younger adults' negative attitudes about ageing in general impacted the observed dementianegative effects. Further research is required to determine whether ageism might be a factor in dementia stigma, and whether educating younger adults about healthy positive ageing as well as dementia may produce more impactful attitude change. Contrary to the 
findings of Blay and Peluso (2010) and Cheng et al. (2011), the fact that a larger number of the young adult sample were educated to university level did not appear to impact levels of stigma, although the research design and methodology employed did not allow for an appropriate investigation of this.

The results showed that for the care-workers, higher levels of burn-out in terms of feelings of reduced personal accomplishment (MBI) were associated with more stigmatising beliefs and knowledge about dementia and less feelings of comfort around people with dementia (DAS subscales). This is consistent with prior research with dementia careworkers demonstrating that higher levels of optimism towards people with dementia was significantly associated with higher levels of personal accomplishment on the MBI (Kokkonen et al., 2014). Implicit stigma was not associated with burnout, which contradicts the findings of Kelly and Barnes-Holmes (2013); although the target population and levels of implicit stigma between the studies differed as Kelly and colleagues reported higher levels of implicit stigma and burnout overall. Care-workers implicit attitudes were dementiapositive, with only a weak non-significant negative bias demonstrated. Perhaps higher levels of implicit stigma would have resulted in an observed association with burnout, but this is largely speculative. More research is required to gather more conclusive evidence in this regard.

Interestingly, depersonalisation on the burnout scale was associated with implicit dementia-positive biases. This was unexpected but further reiterates the necessity for large-sale, well controlled studies examining how burnout might affect stigma. Although the topic of burnout with dementia professionals has not received much attention in the literature, existing studies suggest that moderate to high levels of burnout may be common among care staff (Duffy, Oyebode, \& Allen, 2009), nurses and psychologists (Todd \& Watts, 2005) working in dementia care. Additional research is required to determine the specific factors that may predict burnout, and the extent to which burnout and stigma might affect staff behaviour towards people with dementia (e.g. MacPherson, Eastley, Richards, \& Mian, 1994). Interventions should target supporting dementia care-workers in improving perceptions of competence and satisfaction in their caregiving role (Kokkonen et al., 2014).

Finally, contrary to our hypotheses and unlike the findings of Kelly and Barnes-Holmes (2013), there were no correlations between implicit stigma and measures of depression, anxiety, stress or burnout. This may be due to low overall reported levels of these outcomes, or due to the specificity of the measures utilised. Although factor analysis and convergent validity testing demonstrated the DAS as a useful tool for measuring attitudes towards dementia, the inclusion of a scale designed to measure stigma specifically may be of benefit. Alzheimer's Australia researchers have developed the 31-item Dementia Stigma Questionnaire (DSQ; Phillipson, Magee, Jones, Skladzien, \& Cridland, 2012) which has three subscales of response to dementia diagnosis, avoidance/discrimination, and positive beliefs; and may offer additional insights to the DAS. Similarly, although two meta-analyses have demonstrated the reliability and validity of the IRAP as measure of implicit cognition (Golijani-Moghaddam, Hart, \& Dawson, 2013; Vahey, Nicholson, \& Barnes-Holmes, 2015), the stimuli selected for the current study were largely exploratory and may require adaptation. One option might be to take stimuli directly from validated explicit measures of stigma. The fact that DAS did not correlate IRAP trial-types may suggest that the IRAP did not accurately measure dementia stigma, although it is more likely that the IRAP identified implicit biases not detected by the questionnaire. This finding is common in implicit research 
(Hughes, Barnes-Holmes, \& Vahey, 2012). It would be of benefit to examine relationships between the IRAP and alternative measures of stigma, as suggested above.

There are several limitations to this study that should be noted. Firstly, the small sample size and convenience sampling approach limit the generalisability of the results. It would be interesting for future research to replicate the study design but to use a larger sample and reduce sampling bias. Secondly, the study was cross-sectional and correlational so temporal or causal relationships between variables cannot be inferred. Future studies should include a controlled design to compare groups and examine relationships between the relevant variables longitudinally. Finally, we observed quite a high attrition rate for care-workers, which occurred because of individuals failing to meet the pre-specified latency criteria on the IRAP. This may have been a result of variables including the difficulty of the stimuli used, the instructions provided, or difficulty with using a computer. Research has often reported that older adults or those in certain professions are less likely to have as much engagement with computers as younger adults, and as a result, experience higher rates of anxiety than younger adults in relation to attending to computer tasks (Laguna \& Babcock, 1997). Future studies should conduct pilot testing with such samples to ensure that necessary supports are in place to facilitate progression through the IRAP programme.

\section{Conclusions}

Overall the results are consistent with prior research, and demonstrated that stigma exists towards adults with dementia, that experience working with people with dementia may reduce stigma, and that higher levels of burnout may be related to higher levels of stigmatising attitudes. These findings have implications for the development of training, education and staff-support strategies to reduce stigma towards people with dementia. The reduction of stigma is important to remove barriers to early diagnosis, intervention and community support. Further considerations related to selection of appropriate outcome measures should be made in order to advance our understanding of explicit and implicit stigma towards people with dementia.

\section{Declarations of Conflicting Interests}

The author(s) declared no potential conflicts of interest with respect to the research, authorship, and/or publication of this article.

\section{Funding}

The author(s) declared the following potential conflicts of interest with respect to the research, authorship, and/or publication of this article: all research procedures were conducted in accord with current ethical practices in the field of psychology.

\section{ORCID iD}

Michelle Kelly (D) http://orcid.org/0000-0001-6159-6707

\section{References}

Astrom, S., Nilsson, M., Norberg, A., Sandman, P., \& Winblad, B., (1991). Staff burnout in dementia care - Relations to empathy and attitudes. International Journal of Nursing Studies, 28, 65-75. 
Bamford, C., Lamont, S., Eccles, M., Robinson, L., May, C., \& Bond, J. (2004). Disclosing a diagnosis of dementia: A systematic review. International Journal of Geriatric Psychiatry, 19(2), 151-169.

Barnes-Holmes, D., Barnes-Holmes, Y., Power, P., Hayden, E., Milne, R., \& Stewart, I. (2006). Do you really know what you believe? Developing the Implicit Relational Assessment Procedure (IRAP) as a direct measure of implicit beliefs. The Irish Psychologist, 32, 169-177.

Barnes-Holmes, D., Barnes-Holmes, Y., Stewart, I., \& Boles, S. (2010). A sketch of the implicit relational assessment procedure (IRAP) and the relational elaboration and coherence (REC) model. The Psychological Record, 60, 527-542.

Barnes-Holmes, D., Murtagh, L., Barnes-Holmes, Y., \& Stewart, I. (2010). Using the Implicit Association Test and the Implicit Relational Assessment Procedure to measure attitudes towards meat and vegetables in vegetarians and meat-eaters. The Psychological Record, 60, 287-306.

Batsch, N. L., \& Mittelman, M. (2012). World Alzheimer Report: Overcoming the stigma of dementia. London: Alzheimer's Disease International.

Benbow, S. M., \& Jolley, D. (2012). Dementia: Stigma and its effects. Neurodegenerative Disease Management, 2(2), 165-172.

Bender, M. P., \& Cheston, R. (1997). Inhabitants of a lost kingdom: A model of the subjective experiences of dementia. Ageing \& Society, 17(5), 513-532.

Blay, S. L., \& Peluso, E. T. P. (2010). Public stigma: The community's tolerance of Alzheimer disease. American Journal of Geriatric Psychiatry, 18, 163-171.

Boustani, M., Perkins, A. J., Monahan, P., Fox, C., Watson, L., Hopkins, J., \& Hendrie, H. C. (2008). Measuring primary care patients' attitudes about dementia screening. International Journal of Geriatric Psychiatry, 23(8), 812-820.

Brodaty, H., Draper, B., \& Low, L. F. (2003). Nursing home staff attitudes towards residents with dementia: strain and satisfaction with work. Journal of advanced nursing, 44(6), 583-590.

Burgener, S. C., \& Berger, B. (2008). Measuring perceived stigma in persons with progressive neurological disease Alzheimer's dementia and Parkinson's disease. Dementia, 7(1), 31-53.

Cahill, S., O'Shea, E., \& Pierce, M. (2012). Creating excellence in dementia care: A research review for Ireland's national dementia strategy. Trinity College Dublin.

Cheng, S. T., Lam, L. C., Chan, L. C., Law, A. C., Fung, A. W., Chan, W. C., ... Chan, W. M. (2011). The effects of exposure to scenarios about dementia on stigma and attitudes toward dementia care in a Chinese community. International Psychogeriatrics, 23(09), 1433-1441.

Chirwa, M. L., Greeff, M., Kohi, T. W., Naidoo, J. R., Makoae, L. N., Dlamini, P. S.,\& Holzemer, W. L. (2009). HIV stigma and nurse job satisfaction in five African countries. Journal of the Association of Nurses in AIDS Care, 20(1), 14-21.

Clafferty, R. A., McCabe, E., \& Brown, K. W. (2001). Conspiracy of silence? Telling patients with schizophrenia their diagnosis. The Psychiatrist, 25(9), 336-339.

Clare, L. (2003). Managing threats to self: Awareness in early stage Alzheimer's disease. Social Science \& Medicine, 57(6), 1017-1029.

Corrigan, P. W., \& Watson, A. C. (2002). Understanding the impact of stigma on people with mental illness. World Psychiatry, 1(1), 16-20.

Corrigan, P. W., River, L. P., Lundin, R. K., Wasowski, K. U., Campion, J., Mathisen, J., ... Kubiak, M. A. (2000). Stigmatizing attributions about mental illness. Journal of Community Psychology, 28(1), 91-102.

Crisp, A., Gelder, M., Goddard, E., \& Meltzer, H. (2005). Stigmatization of people with mental illnesses: a follow-up study within the Changing Minds campaign of the Royal College of Psychiatrists. World psychiatry, 4(2), 106-113.

Crawford, J. R., \& Henry, J. D. (2003). The Depression Anxiety Stress Scales (DASS): Normative data and latent structure in a large non-clinical sample. British journal of clinical psychology, 42(2), 111-131. 
Cullen, C., \& Barnes-Holmes, D. (2008). Implicit pride and prejudice: A heterosexual phenomenon? In T. G. Morrison \& M. A. Morrison (Eds.), Modern prejudice. (pp. 195-223). New York: Nova Science.

Cullen, C., Barnes-Holmes, D., Barnes-Holmes, Y., \& Stewart, I. (2009). The Implicit Relational Assessment Procedure (IRAP) and the malleability of ageist attitudes. The Psychological Record, 59, 591-620.

De Houwer, J. (2006). What are implicit measures and why are we using them? In R. Wiers \& A. Stacy (Eds.), Handbook of implicit cognition and addiction. New York: Sage.

Dinos, S., Stevens, S., Serfaty, M., Weich, S., \& King, M. (2004). Stigma: The feelings and experiences of 46 people with mental illness. The British Journal of Psychiatry, 184(2), 176-181. DOI: 10.1192/bjp.184.2.176.

Draper, B., Snowdon, J., Meares, S., Turner, J., Gonski, P., McMinn, B., ... Luscombe, G. (2000). Case-controlled study of nursing home residents referred for treatment of vocally disruptive behavior. International Psychogeriatrics, 12(03), 333-344.

Duffy, B., Oyebode, J. R., \& Allen, J. (2009). Burnout among care staff for older adults with dementia: The role of reciprocity, self-efficacy and organizational factors. Dementia, 8(4), 515-554.

Ewalds-Kvist, B., Högberg, T., \& Lützén, K. (2013). Impact of gender and age on attitudes towards mental illness in Sweden. Nordic Journal of Psychiatry, 67(5), 360-368.

Fratiglioni, L., Launer, L. J., Andersen, K., Breteler, M. M., Copeland, J. R., Dartigues, J. F., ... Hofman, A. (2000). Incidence of dementia and major subtypes in Europe: A collaborative study of population-based cohorts. Neurologic Diseases in the Elderly Research Group. Neurology, $54, \mathrm{~S} 10-\mathrm{S} 15$.

George, D. R., Stuckey, H. L., \& Whitehead, M. M. (2014). How a creative storytelling intervention can improve medical student attitude towards persons with dementia: A mixed methods study. Dementia, 13(3), 318-329.

Golijani-Moghaddam, N., Hart, A., \& Dawson, D. L. (2013). The Implicit Relational Assessment Procedure: Emerging reliability and validity data. Journal of Contextual Behavioral Science, $2,105-119$.

Grand, J. H., Caspar, S., \& MacDonald, S. W. (2011). Clinical features and multidisciplinary approaches to dementia care. Journal of Multidisciplinary Healthcare, 4, 125-147. DOI: 10.2147/ JMDH.S17773.

Greene, J. D., Hodges, J. R., \& Baddeley, A. D. (1995). Autobiographical memory and executive function in early dementia of Alzheimer type. Neuropsychologia, 33(12), 1647-1670.

Greenwald, A. G., McGhee, D. E., \& Schwartz, J. L. (1998). Measuring individual differences in implicit cognition: The implicit association test. Journal of Personality and Social Psychology, 74(6), 1464.

Greenwald, A. G., Nosek, B. A., \& Banaji, M. R. (2003). Understanding and using the Implicit Association Test: I. An improved scoring algorithm. Journal of Personality and Social Psychology, 85(2), 197-216.

Hastings, R. (2009). An exploration into family caregivers' experiences of caring for a person with dementia (Unpublished thesis). MSW, Department of Social Studies, Trinity College Dublin.

Herrmann, L. K., Welter, E., Leverenz, J., Lerner, A. J., Udelson, N., Kanetsky, C., \& Sajatovic, M. (2017). A systematic review of dementia-related stigma research: can we move the stigma dial?. The American Journal of Geriatric Psychiatry, 26(3), 316-331.

Hughes, S., Barnes-Holmes, D., \& De Houwer, J. (2011). The dominance of associative theorising in implicit attitude research: Propositional and behavioral alternatives. The Psychological Record, 61, 465-496.

Hughes, S., Barnes-Holmes, D., \& Vahey, N. (2012). Holding on to our functional roots when exploring new intellectual islands: A voyage through implicit cognition research. Journal of Contextual Behavioral Science, 1(1-2), 17-38. DOI: 10.1016/j.jcbs.2012.09.003. 
Hussey, I., Barnes-Holmes, D., \& Barnes-Holmes, Y. (2015). From Relational Frame Theory to implicit attitudes and back again: Clarifying the link between RFT and IRAP research. Current Opinions in Psychology, 2, 11-15.

Iliffe, S., D., Lepeliere, J., van Hout, H., Kenny, H., Lewis, A., \& Vernooij-Dassen, M. (2005). Understanding obstacles to the recognition of and response to dementia in different European countries: A modified focus group approach using multinational, multi-disciplinary expert groups. Aging and Mental Health, 9(1), 1-6.

Jessen, F., Amariglio, R. E. V., Boxtel, M., Breteler, M., Ceccaldi, M., Chételat, G., ..., Glodzik, L. (2014). A conceptual framework for research on subjective cognitive decline in preclinical Alzheimer's disease. Alzheimer's \& Dementia, 10(6), 844-852.

Jorm, A. F. (2000). Mental health literacy: Public knowledge and beliefs about. British Journal of Psychiatry, 177, 396-401.

Kada, S., Nygaard, H. A., Mukesh, B. N., \& Geitung, J. T. (2009). Staff attitudes towards institutionalised dementia residents. Journal of Clinical Nursing, 18, 2382-2392.

Katz, I. R. (1998). Diagnosis and treatment of depression in patients with Alzheimer's disease and other dementias. The Journal of Clinical Psychiatry, 59(Suppl. 9), 38-44.

Kelly, A., \& Barnes-Holmes, D. (2013). Implicit attitudes towards children with autism versus normally developing children as predictors of professional burnout and psychopathology. Research in Developmental Disabilities, 34(1), 17-28.

Kokkonen, T. M., Cheston, R. I., Dallos, R., \& Smart, C. A. (2014). Attachment and coping of dementia care staff: The role of staff attachment style, geriatric nursing self-efficacy, and approaches to dementia in burnout. Dementia (London), 13(4), 544-568. DOI: 10.1177/ 1471301213479469 .

Laguna, K., \& Babcock, R. L. (1997). Computer anxiety in young and older adults: Implications for human-computer interactions in older populations. Computers in Human Behavior, 13(3), 317-326.

Lobo, A., Saz, P., Marcos, G., Día, J. L., \& De-la-Cámara, C. (1995). The prevalence of dementia and depression in the elderly community in a southern European population. The Zaragoza Study. Archives of General Psychiatry, 52(6), 497-506.

Lovibond, P. F., \& Lovibond, S. H. (1995). The structure of negative emotional states: Comparison of the Depression Anxiety Stress Scales (DASS) with the Beck Depression and Anxiety Inventories. Behaviour research and therapy, 33(3), 335-343.

MacPherson, R., Eastley, R. J., Richards, H., \& Mian, I. H. (1994). Psychological distress among workers caring for the elderly. International Journal of Geriatric Psychiatry, 9, 381-386.

Maslach, C., \& Jackson, S. E. (1981). The measurement of experienced burnout. Journal of Occupational Behavior, 2, 99-113. doi10.1002/job.4030020205.

McParland, P., Devine, P., Innes, A., \& Gayle, V. (2012). Dementia knowledge and attitudes of the general public in Northern Ireland: An analysis of national survey data. International Psychogeriatrics, 24(10), 1600-1613.

Mitchell, A. J. (2007). Reluctance to disclose difficult diagnoses: A narrative review comparing communication by psychiatrists and oncologists. Supportive Care in Cancer, 15(7), 819-828.

Moniz-Cook, E., Agar, S., Gibson, G., Win, T., \& Wang, M. (1998). A preliminary study of the effects of early intervention with people with dementia and their families in a memory clinic. Aging \& Mental Health, 2(3), 199-211.

Moyle, W., Murfield, J. E., Griffiths, S. G., \& Venturato, L. (2011). Care staff attitudes and experiences of working with older people with dementia. Australasian Journal on Ageing, 30(4), 186-190.

Murphy, C., Hussey, T., Barnes-Holmes, D., \& Kelly, M. E. (2015). The Implicit Relational Assessment procedure (IRAP) and attractiveness bias. Journal of Contextual Behavioral Science, 4(4), 292-299. 
Murphy, C., MacCarthaigh, S., \& Barnes-Holmes, D. (2014). Implicit relational assessment procedure and attractiveness bias: Directionality of bias and influence of gender of participants. International Journal of Psychology and Psychological Therapy, 14(3), 333-351.

Nicholson, E., \& Barnes-Holmes, D. (2012). The Implicit Relational Assessment Procedure (IRAP) as a measure of spider fear. The Psychological Record, 62(2), 263-277.

O'Connor, M. L., \& McFadden, S. H. (2010). Development and psychometric validation of the Dementia Attitudes Scale. International Journal of Alzheimer's Disease, 2010, 1-10.

Ólafsdóttir, M., Foldevi, M., \& Marcusson, J. (2001). Dementia in primary care: why the low detection rate?. Scandinavian journal of primary health care, 19(3), 194-198.

Park, S., Kim, M. J., Cho, M. J., \& Lee, J. Y. (2015). Factors affecting stigma toward suicide and depression: A Korean nationwide study. International Journal of Social Psychiatry, 61(8), 811-817.

Peris, T. S., Teachman, B. A., \& Nosek, B. A. (2008). Implicit and explicit stigma of mental illness: Links to clinical care. Journal of Nervous and Mental Disease, 196, 752-760.

Phillipson, L., Magee, D. C., Jones, S., Skladzien, D. E., \& Cridland, E. (2012). Exploring dementia and stigma beliefs. Alzheimer's Australia.

Prince, M., Wimo, A., Guerchet, M., Ali, G. C., Wu, Y. T., \& Prina, M. (2015). World Alzheimer Report 2015. The global impact of dementia. London, UK: Alzheimer's Disease International.

Richardson, B., Kitchen, G., \& Livingston, G. (2002). The effect of education on knowledge and management of elder abuse: A randomized controlled trial. Age and Ageing, 31, 335-341.

Roddy, S., Stewart, I., \& Barnes-Holmes, D. (2010). Anti-fat, pro-slim, or both? Using two reaction time based measures to assess implicit attitudes to the slim and overweight. Journal of Health Psychology, 15, 416-425.

Rodney, V. (2000). Nurse stress associated with aggression in people with dementia: Its relationship to hardiness, cognitive appraisal and coping. Journal of Advanced Nursing, 31(1), 172-180.

Rüsch, N., Angermeyer, M. C., \& Corrigan, P. W. (2005). Mental illness stigma: Concepts, consequences, and initiatives to reduce stigma. European Psychiatry, 20(8), 529-539.

Scerri, A., \& Scerri, C. (2013). Nursing students' knowledge and attitudes towards dementia-A questionnaire survey. Nurse Education Today, 33(9), 962-968.

Scholl, J. M., \& Sabat, S. R. (2008). Stereotypes, stereotype threat and ageing: Implications for the understanding and treatment of people with Alzheimer's disease. Ageing and Society, 28(01), 103-130.

Spector, A., Thorgrimsen, L., Woods, B. O. B., Royan, L., Davies, S., Butterworth, H. M., \& Orrell, M. (2003). Efficacy of an evidence-based cognitive stimulation therapy programme for people with dementia. The British Journal of Psychiatry, 183(3), 248-254.

Taris, T. W., Schreurs, P. J., \& Schaufeli, W. B. (1999). Construct validity of the Maslach Burnout Inventory-General Survey: A two-sample examination of its factor structure and correlates. Work \& Stress, 13(3), 223-237.

Teri, L., Logsdon, R. G., Uomoto, J., \& McCurry, S. M. (1997). Behavioral treatment of depression in dementia patients: A controlled clinical trial. The Journals of Gerontology Series B: Psychological Sciences and Social Sciences, 52(4), 159-166.

Todd, S. J., \& Watts, S. C. (2005). Staff responses to challenging behaviour shown by people with dementia: An application of an attributional-emotional model of helping behaviour. Aging and Mental Health, 9(1), 71-81.

Vahey, N. A., Nicholson, E., \& Barnes-Holmes, D. (2015). A meta-analysis of criterion effects for the Implicit Relational Assessment Procedure (IRAP) in the clinical domain. Journal of Behavior Therapy and Experimental Psychiatry, 48, 59-65. DOI: 10.1016/j.jbtep.2015.01.004.

Werner, P. (2005). Lay perceptions about mental health: Where is age and where is Alzheimer's disease? International Psychogeriatrics, 17(03), 371-382.

Werner, P., \& Davidson, M. (2004). Emotional reactions of lay persons to someone with Alzheimer's disease. International Journal of Geriatric Psychiatry, 19(4), 391-397.

Winslow, B. T., Onysko, M. K., Stob, C. M., \& Hazlewood, K. A. (2011). Treatment of Alzheimer disease. American Family Physician, 83(12), 1403-1412. 
Alan Kane has an Honours Degree and a Research Master's (MRes) in Psychology from Maynooth University; and will graduate with a Master's in Applied Behaviour Analysis from National University of Ireland Galway in September 2018. He completed this research as part of his MRes in Psychology.

Carol Murphy has an Honours Degree in Psychology and a PhD in Applied Behaviour Analysis from Maynooth University. She is currently a lecturer in Psychology in Maynooth University and co-supervised this research.

Michelle Kelly has an Honours Degree in Psychology and a Doctorate in Applied Behaviour Analysis from Maynooth University. She is currently a lecturer in Psychology in the National College of Ireland and was the primary supervisor and co-author on this research. 